\title{
ИДЕНТИФИКАЦИЯ ФИЛЬТРАЦИОННЫХ ПОТОКОВ В ПРОЦЕССЕ ГИДРОДИНАМИЧЕСКИХ ИССЛЕДОВАНИЙ ГОРИЗОНТАЛЬНЫХ СКВАЖИН С ТРЕЩИНАМИ ГИДРОРАЗРЫВА ПЛАСТА
}

\author{
Сергеев Виктор Леонидович',
}

SergeevVL@ignd.tpu.ru

\author{
Донг Ван Хоанг', \\ hoang.tpu@gmail.com.
1 Национальный исследовательский Томский политехнический университет, Россия, 634050, г. Томск, пр. Ленина, 30.

Актуальность работы вызвана необходимостью создания методов оперативной интерпретации результатов нестационарных исследований по восстановлению забойного давления горизонтальных скважин с трещинами гидроразрыва пласта, повышения достоверности оценок параметров нефтяных пластов и сокращения времени простоя скважин.

Целью исследования является разработка моделей и алгоритмов идентификации фильтрационных потоков для определения фильтрационных параметров и пластового давления в процессе нестационарных исследований горизонтальных скважин с трещинами гидроразрыва пласта.

Методы основаны на использовании результатов гидродинамических исследований скважин с трещинами гидроразрыва пласта, системного анализа, моделирования систем с учетом дополнительной информации и экспертных оценок, оптимизации функций, линейной алгебры. Решение задачи идентификации фильтрационных потоков проводилось с использованием интегрированных систем моделей забойного давления с нестационарными параметрами, с учетом дополнительных сведений и экспертных оценок проницаемости пласта и пластового давления. Апробация моделей и алгоритмов идентификации потоков и определения параметров пласта и трещины проводилась с использованием промысловых данных нестационарных исследований горизонтальных скважин нефтяных месторождений по восстановлению забойного давления с трещинами гидроразрыва пласта с использованием программного комплекса Saphir.

Результаты. На примере обработки результатов нестационарных исследований по восстановлению забойного давления горизонтальных скважин нефтяного месторождения показано, что предложенные модели и алгоритмы идентификации фильтрационных потоков позволяют определять эффективную длину горизонтального участка ствола скважины, латеральную проницаемости, пластовое давление и скин-фактор в процессе проведения исследований, в условиях частичного либо полного отсутствия на кривой восстановления давления участка позднего радиального потока, значительно сократить время простоя скважин.

\section{Ключевые слова:}

Нестационарные исследования, фильтрационные потоки, идентификация, адаптация,

забойное давление, горизонтальные скважины, нефтяные пласты, трещины гидроразрыва пласта.

\section{Введение}

В настоящее время актуальной является проблема интерпретации результатов нестационарных исследований горизонтальных скважин по восстановлению забойного давления с трещинами гидроразрыва пласта (ГРП). Отметим, что использование традиционных графоаналитических методов интерпретации, основанных на исследовании кривой восстановления забойного давления, вызывает значительные трудности, связанные с отсутствием участков раннего и позднего радиальных потоков, искажениями на этих участках забойного давления. Так, например, для получения полноценных кривых восстановления забойного давления горизонтальных скважин с трещинами ГРП с участком позднего радиального потока требуется проведение достаточно продолжительных исследований, порядка 1000 часов и более. Интерпретация таких кривых восстановления забойного давления требует привлечения квалифицированных интерпретаторов, снижает оперативность получения необходимой для контроля и управления работой скважин информации, приводит к значительным материальным затратам [1-10].

В этой связи актуальна задача разработки моделей и алгоритмов оперативной обработки ре- зультатов нестационарных исследований горизонтальных скважин с трещинами ГРП в процессе проведения гидродинамических исследований в условиях частичного либо полного отсутствия на кривой восстановления давления участка позднего радиального потока. Решение данной проблемы для скважин без ГРП было рассмотрено в работе [11], где задача идентификации позднего радиального потока решалась с использованием адаптивного метода обработки результатов нестационарных исследований горизонтальных скважин и уравнения Вольтерра 1-го рода. Показано, что разработанные модели и алгоритмы идентификации позволяют оперативно, в процессе проведения нестационарных исследований, обрабатывать короткие недовосстановленные кривые забойного давления, определять параметры пласта и время завершения исследований.

В данной работе рассматривается задача оперативной идентификации фильтрационных потоков в процессе проведения нестационарных исследований горизонтальных скважин с трещинами ГРП с использованием интегрированной системы моделей забойного давления с нестационарными параметрами, с корректировкой дополнительной информации о проницаемости пласта. Предлагается 
новый алгоритм решения оптимизационных задач по определению параметров интегрированных моделей забойного давления и параметров корректировки дополнительной информации и экспертных оценок.

\section{Модели и алгоритмы идентификации фильтрационных потоков}

В основе задачи идентификации фильтрационных потоков по кривой восстановления забойного давления горизонтальной скважины с трещинами ГРП использованы интегрированные системы моделей с нестационарными параметрами $\alpha_{n}=\alpha\left(t_{n}\right)$, с использованием дополнительной информации вида:

$$
\left\{\begin{array}{l}
P_{3, j}^{*}\left(t_{n}\right)= \\
=P_{3, J}\left(t_{0}\right)+f_{\mathrm{o}, j}\left(t_{n}, \alpha_{n, j}\right)+\xi\left(t_{n}\right), t_{n} \in\left(t_{\mathrm{r}, j} t_{\mathrm{\kappa}, j}\right), \\
h_{j, k} \cdot \bar{z}_{j, \mathrm{\kappa}}=f_{a, j, \mathrm{~K}}\left(t_{n}, \alpha_{n, j}\right)+\eta_{n}, j=\overline{1,4}, k=\overline{1, l},
\end{array}\right.
$$

где $P_{3, j}^{*}\left(t_{n}\right), P_{3, J}\left(t_{0}\right)$ - фактические значения забойного давления, полученные в моменты времени $t_{n}$ и $t_{0}$; $f_{o, j}\left(t_{n}, \alpha_{n, j}\right)$ - значения забойного давления, вычисленные на основе соответствующей модели фильт- рационного потока с номером $j$ (табл. 1$) ; \bar{z}_{j, k}-$ фактические значения дополнительных априорных данных и экспертных оценок параметров нефтяных пластов; $f_{o, j, k}\left(t_{n}, \alpha_{n, j}\right)$ - значения дополнительных данных и экспертных оценок, полученные на основе модели; $h_{j, k}$ - параметры корректировки неточно заданных дополнительных данных и экспертных оценок параметров пласта; $t_{\mathrm{H}, j}, t_{\mathrm{k}, j}-$ момен- $^{-}$ ты времени начала и завершения фильтрационного потока с номером $j ; \xi_{n}, \eta_{n}$ - случайные величины, связанные с погрешностью измерений забойного давления и ошибками задания экспертных оценок и других неконтролируемых факторов.

Модели фильтрационных потоков горизонтальных скважин с трещинами ГРП приведены в табл. 1. На рис. 1 представлен диагностический график фильтрационных потоков в билогарифмических координатах.

Решение задачи идентификации фильтрационных потоков рассмотрим на примере интегрированной системы моделей забойного давления позднего радиального потока с учетом дополнительной априорной информации и экспертных оценок о латеральной проницаемости $\bar{k}_{r, n}[11]$

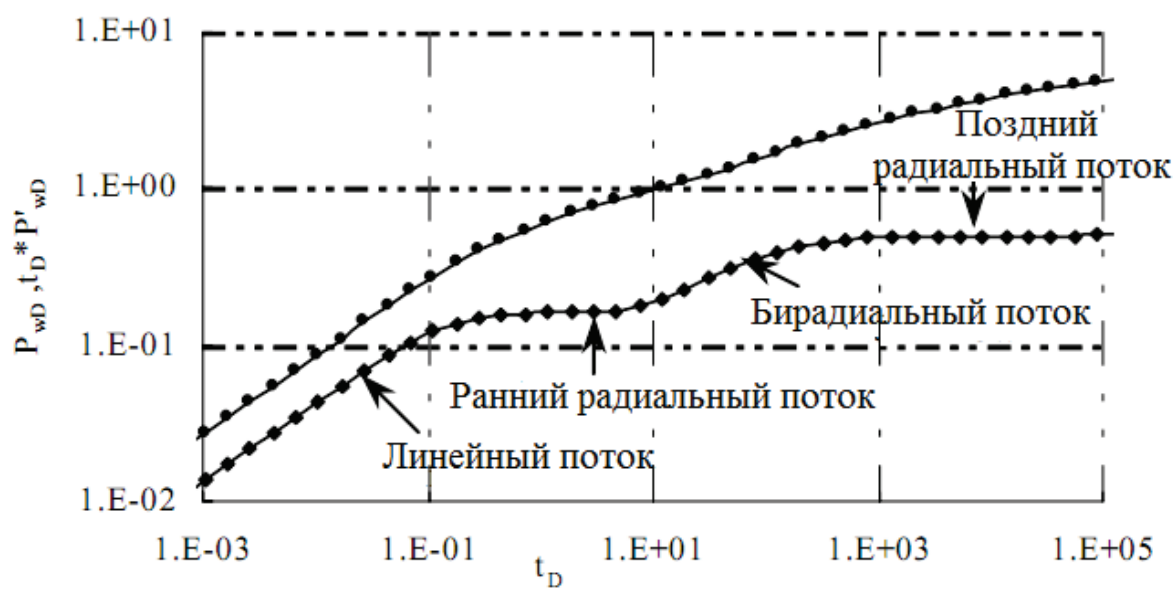

Pис. 1. Диагностический график потоков в билогарифмических координатах [12]

Fig. 1. Diagnostic plot of flow regimes in log-log scales [12]

Таблица 1. Модели фильтращионных потоков для горизонтальных скважин с трещинами ГРП [12-16]

Table 1. Models of filtration flow regimes of horizontal wells with hydraulic fractures [12-16]

\begin{tabular}{|c|c|c|}
\hline $\begin{array}{l}\text { Фильтрационные потоки } \\
\text { Filtration flow regimes }\end{array}$ & $\begin{array}{l}\text { Уравнение } \\
\text { Equation }\end{array}$ & $\begin{array}{l}\text { Дополнительная информация } \\
\text { Additional information }\end{array}$ \\
\hline $\begin{array}{l}\text { Линейный } \\
\text { Linear flow }\end{array}$ & $\Delta P_{3}=\frac{C_{s l} q B}{h}\left(\frac{\mu}{\phi c_{t} k_{y} x_{f}}\right)^{0,5} \sqrt{t}$ & $\begin{array}{l}x_{f}-\text { полудлина трещин } \\
\text { half fracture length }\end{array}$ \\
\hline $\begin{array}{l}\text { Ранний радиальный } \\
\text { First radial }\end{array}$ & $\Delta P_{3}=\frac{C_{s f r} q \mu B}{N_{f} h k_{r}} \ln \left(\frac{2,25 k_{r} t}{\phi C_{t} \mu r_{\text {mp }}^{2}}\right)$ & $\begin{array}{l}N_{f}-\text { количество трещин } \\
\text { number of fractures }\end{array}$ \\
\hline $\begin{array}{l}\text { Бирадиальный } \\
\text { Biradial flow }\end{array}$ & $\Delta P_{3}=\frac{C_{s b r} q \mu B}{k_{y} h}\left(\frac{4}{L^{2} \phi \mu c_{t}}\right)^{0,36} t^{0,36}$ & $\begin{array}{l}L \text { - длина горизонтального ствола } \\
\text { length of horizontal wellbore }\end{array}$ \\
\hline $\begin{array}{l}\text { Поздний радиальный } \\
\text { Late radial }\end{array}$ & $\Delta P_{3}=\frac{C_{\text {spr }} q \mu B}{h k_{r}} \ln \left(\frac{2,25 k_{r} t}{\phi \mu c_{t} r_{\text {mp }}^{2}}\right)$ & $\begin{array}{c}P_{\text {пл }}-\text { пластовое давление } / \text { reservoir pressure } \\
k_{r}=\sqrt{k_{x} k_{y}}-\text { латеральная проницаемость } \\
\text { lateral permeability }\end{array}$ \\
\hline
\end{tabular}




$$
\left\{\begin{array}{l}
P_{3}^{*}\left(t_{n}\right)=P_{3}\left(t_{0}\right)+f\left(b_{n}, t_{n}\right)\left(\alpha_{1, n}+\alpha_{2 n} \ln t_{n}\right)+\xi_{n}, \\
h_{k, n} \cdot \bar{\alpha}_{2}=\alpha_{2, n}+\eta_{n}, t_{n}=\overline{t_{n .6 p}, t_{n k}},
\end{array}\right.
$$

где $f\left(b_{n}, t_{n}\right) \rightarrow 1$ при $t_{n} \rightarrow \infty$ - поправочная функция с переменным, зависящим от времени $t_{n}$ с параметром $b_{n} ; \alpha_{1 . n}=\frac{C_{s} q_{0} \mu B}{4 \pi k_{r, n} h} \ln \left(\frac{2,25 k_{r, n}}{\phi \mu r_{\text {пр, }, n}^{2}}\right) ; \alpha_{2, n}=\frac{C_{s} q_{0} \mu B}{4 \pi k_{r, n} h} ;$ $\bar{\alpha}_{2}=\frac{C_{s} q_{0} \mu B}{4 \pi \overline{k_{r}} h} ; k_{r, n}-$ латеральная проницаемость; $q_{0}$ - дебит скважины, см ${ }^{3} / \mathrm{c} ; \phi$ - пористость, доля; $\mu$ - вязкость нефти, сПз; $r_{\text {пр, }}$ - приведенный радиус скважины, см; $B$ - объемный коэффициент, $\mathrm{M}^{3} / \mathrm{M}^{3} ; h$ - толщина пласта, см; $\overline{k_{r}}-$ дополнительные данные и экспертные оценки латеральной проницаемости; $C_{s}$ - константа, зависящая от используемой системы единиц.

Особенность модели (2) заключается в том, что момент времени завершения исследований $t_{n k}$ является неизвестной величиной и определяется наряду с неизвестными параметрами $\alpha_{1, n}, \alpha_{2, n}, b_{n}, h_{k, n}$ (2) в процессе проведения гидродинамических исследований.

Решение задачи определения параметров модели (2), представленной для удобства в матричном виде,

$$
\left\{\begin{array}{l}
\operatorname{deltaP}_{3, n}^{*}=F\left(b_{n}\right) \cdot \alpha_{n}+\xi_{n}, \\
h_{k, n} \cdot \bar{\alpha}=\Gamma \cdot \alpha_{n}+\Gamma \cdot \eta_{n}, n=1, n_{k}
\end{array}\right.
$$

по аналогии с [17] сводится к решению двух одномерных оптимизационных задач по определению параметра $b_{n}$ модели поправочной функции $f\left(b_{n}, t_{n}\right)$ и параметра корректировки $h_{k, n}$ вида:

$$
\begin{gathered}
b_{n}^{*}\left(h_{k, n}\right)= \\
\left.=\underset{b_{n}}{\arg \min }\left(\| \operatorname{deltaP_{3,n}^{*}}-F_{n}\left(b_{n}\right) \cdot \boldsymbol{\alpha}_{n}^{*}\left(b_{n}, h_{k, n}\right)\right) \|_{W_{n}}^{2}\right), \\
\left.h_{k, n}^{*}=\arg \min _{h_{k, n}}\left(\operatorname{delta} \| P_{3, n}^{*}-F_{n}\left(b_{n}^{*}\right) \cdot \alpha_{n}^{*}\left(b_{n}^{*}, h_{k, n}\right)\right) \|_{W_{n}}^{2}\right),
\end{gathered}
$$

где параметры $\alpha_{n}=\left(\alpha_{1, n}, \alpha_{1, n}\right)^{T}$ (3) определяются из решения системы линейных алгебраических уравнений

$$
\begin{aligned}
& \left(F\left(b_{n}\right)^{T} W_{n} F\left(b_{n}\right)+h_{p} I\right) \alpha_{n}= \\
& =F\left(b_{n}\right)^{T} W_{n} \Delta P_{3, n}^{*}+h_{k, n} \cdot \bar{\alpha} .
\end{aligned}
$$

В (3)-(6) приняты обозначения: delta $P_{3, n}^{*}=$ $=\left(P_{3}^{*}\left(t_{i}\right)-P_{3}^{*}\left(t_{0}\right), i=\overline{1, n}\right)^{T}, \quad \bar{\alpha}=\left(0, \bar{\alpha}_{2}\right)^{T}-\alpha_{n}=\left(\alpha_{1, n}, \alpha_{2, n}\right)^{T^{T}}-$

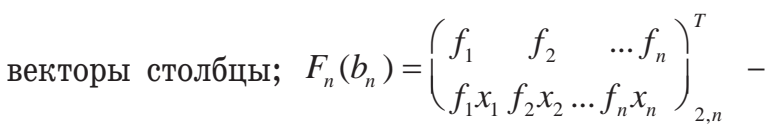
матрица размерности $(2, n)$, в которой $f_{i}=f\left(b_{n}, t_{i}\right)$, $x_{i}=\ln \left(t_{i}\right), i=\overline{1, n} ; W_{n}=\operatorname{diag}\left(w\left(\left(t_{n}-t_{n-i}\right) / h_{3}, i=0, n-1\right)-\right.$ диагональная матрица весовых функций $w\left(\left(t_{n}-t_{n-i}\right) / h_{3}\right)$ с параметром $h_{3}$ для формирования в момент времени $t_{n}$ текущего участка обработки; $h_{p}$ - параметр регуляризации для обеспечения невырожденности матрицы $F\left(b_{n}\right)^{T} W_{n} F\left(b_{n}\right) ; I$ - единич- ная матрица; $\Gamma=\operatorname{diag}(0,1)$ - диагональная матрица; $\|X\|_{W}^{2}=X^{T} W X$ - квадратичная норма вектора $X=\left(x_{1}, x_{2}, \ldots, x_{n}\right) ; \xi_{n}, \eta_{n}$ - векторы случайных величин; $T$ - символ транспонирования.

По аналогии с (1) в (2) имеют место интегрированные системы моделей бирадиального потока (7) $[18,19]$

$$
\left\{\begin{array}{l}
P_{3}^{*}\left(t_{n}\right)=\alpha_{1 b r, n}+\alpha_{2 b r, n} t_{n}^{0,36}+\xi_{n}, \\
h_{k, n} \cdot \bar{\alpha}_{2 b r}=\alpha_{2 b r, n}+\eta_{n}, t_{n}=\overline{t_{0}, t_{n k}}
\end{array}\right.
$$

раннего радиального потока (8)

$$
\left\{\begin{array}{l}
P_{3}^{*}\left(t_{n}\right)=P_{3}\left(t_{0}\right)+\left(\alpha_{1 r, n}+\alpha_{2 r, n} \ln t_{n}\right)+\xi_{n}, \\
h_{k, n} \cdot \bar{\alpha}_{2 r}=\alpha_{2 r, n}+\eta_{n}, t_{n}=\overline{t_{0}, t_{n k}}
\end{array}\right.
$$

и линейного потока (9)

$$
\left\{\begin{array}{l}
P_{3}^{*}\left(t_{n}\right)=\alpha_{1 l, n}+\alpha_{2 l, n} \sqrt{t_{n}}+\xi_{n}, \\
h_{k, n} \cdot \bar{\alpha}_{2 l}=\alpha_{2 l, n}+\eta_{n}, t_{n}=\overline{t_{0}, t_{n k}},
\end{array}\right.
$$

где $\alpha_{1 b r, n}=P_{3}\left(t_{0}\right), \alpha_{2 b r, n}=\frac{C_{s b r} q \mu B}{k_{y} h}\left(\frac{4}{L^{2} \phi \mu c_{t}}\right)^{0,36}$ - параметры модели бирадиального потока; $\alpha_{1 r, n}=\frac{C_{s f r} q \mu B}{N_{f} h k_{r}} \ln \left(\frac{2,25 k_{r}}{\phi C_{t} \mu r_{\text {пр }}^{2}}\right), \alpha_{2 r, n}=\frac{C_{s f r} q \mu B}{N_{f} h k_{r}}$ - параметры модели раннего радиального потока; $\alpha_{1, n}=P_{3}\left(t_{0}\right), \quad \alpha_{2 l, n}=\frac{C_{s l} q B}{h}\left(\frac{\mu}{\phi c_{t} k_{y} x_{f}}\right)^{0,5}-$ параметры модели линейного потока; $k_{y}$ - горизонтальная проницаемость, мД; $C_{s b r}, C_{s f r}, C_{s l}$ - константы, зависящие от используемой системы единиц. Оценки параметров $\alpha_{1 b r}, \alpha_{2 b r}, \alpha_{1 r}, \alpha_{2 r}, \alpha_{1 l}, \alpha_{2 l}$ и параметров корректировки $h_{k}$ определяются по аналогии с (5), (6).

\section{Апробации алгоритмов идентификации фильтрационных потоков}

Результаты апробации моделей и алгоритмов идентификации фильтрационных потоков двух горизонтальных скважин с трещинами ГРП нефтяного месторождения Тюменской области приведены на рис. $2-5$ и в табл. 3. Фактические и восстановленные значения забойного давления скважин № 1 и 2 и их производные, полученные соответственно за 480 и 450 часов гидродинамических исследований, приведены на рис. $2,3$.

На рис. 4 представляются оценки латеральной проницаемости скважин № 1 и 2

$$
k_{r, n}^{*}=\frac{\phi \mu r_{\mathrm{mp}, n}^{2}}{4 \alpha_{2, n}^{*}\left(b_{n}^{*}, h_{k, n}^{*}\right)},
$$

где параметры $b_{n}^{*}, h_{k, n}^{*}$ модели (2) определялись путем решения оптимизационных задач (7), (8) методом дихотомии [20] при постоянных единичных значениях весовой функции в текущем интервале обработке $\left[t_{n}-t_{n-4}\right]$, содержащем пять значений забойного давления $\left(w_{n}\left(\left(t_{n}-t_{n-i}\right) / h_{3}\right)=1\right.$ при $t_{n}-t_{n-i} \leq h_{3}=t_{n}-t_{n-4}$ и $w_{n}\left(\left(t_{n}-t_{n-i}\right) / h_{3}\right)=0$ при $\left.t_{n}-t_{n-i}>h_{3}, i=0, n-1\right)$ и $h_{p}=1$. 
На рис. 5 приведены оценки пластового давления скважин № 1 и 2

$$
P_{\text {пл, },}=f\left(b_{n}^{*}, t_{n}\right)\left(\alpha_{1, n}^{*}+\alpha_{2, n}^{*} \ln T\right),
$$

где $T$ - время восстановления забойного давления до пластового (табл. 2).

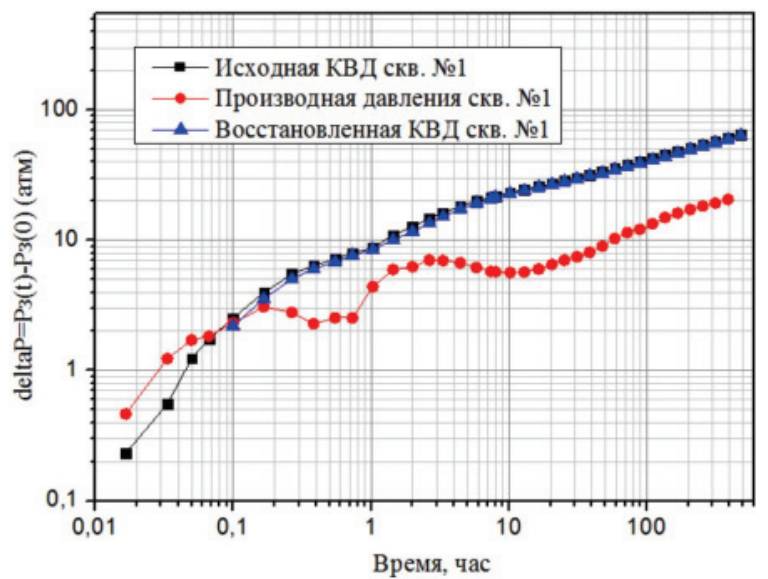

Рис. 2. Кривая восстановления давления скважины № 1 и её производная

Fig. 2. Pressure buildup curve of well 1 and its derivative

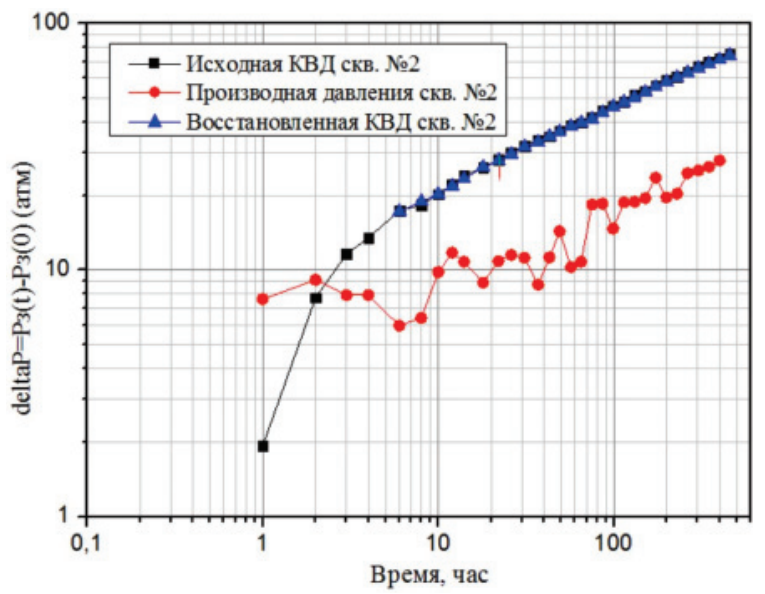

Рис.3. Кривая восстановления давления скважины № 2 и её производная

Fig. 3. Pressure buildup curve of well 2 and its derivative

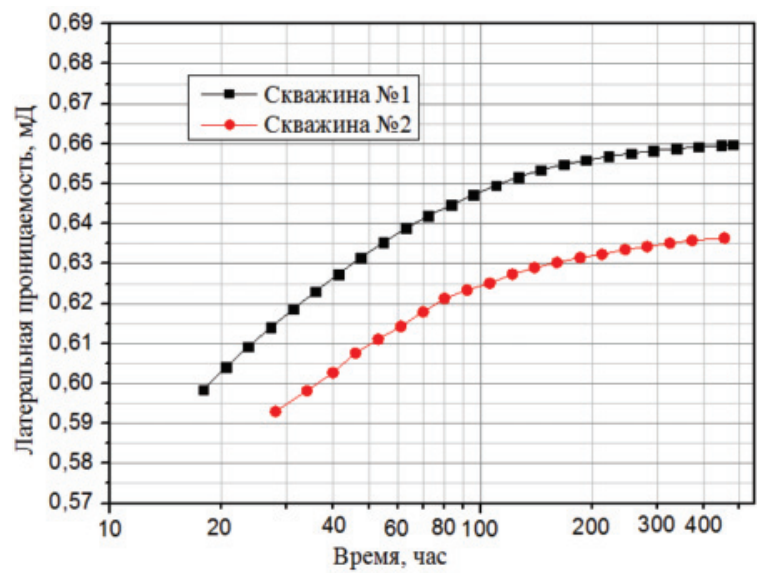

Puс. 4. Оиенки латеральной проницаелости скважины № 1 и 2

Fig. 4. Estimates of lateral permeability of wells 1 and 2

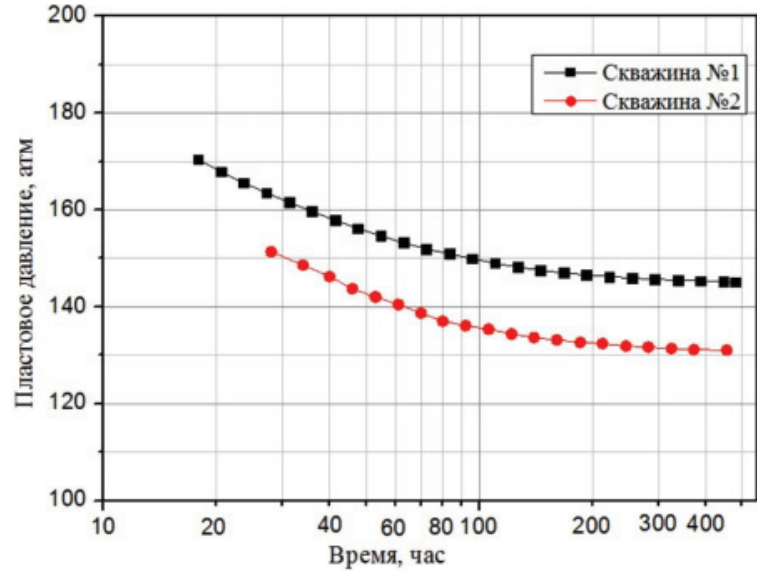

Pис. 5. Оценки пластового давления скважины № 1 и 2

Fig. 5. Estimates of reservoir pressure of wells 1 and 2

В табл. 2 приведены промысловые данные и дополнительные сведения для скважин № 1 и 2.

Таблица 2. Промысловые данные и дополнительные сведения по скважинам № 1 и 2

Table 2. $\quad$ Initial and additional a priori data of wells 1 and 2

\begin{tabular}{|c|c|c|}
\hline \multirow{2}{*}{$\begin{array}{c}\text { Промысловые данные и дополнительные } \\
\text { сведения параметров пласта и скважин } \\
\text { Field data and addition data of reservoir } \\
\text { and well parameters }\end{array}$} & \multicolumn{2}{|c|}{ Скважина/Well } \\
\hline & 1 & 2 \\
\hline $\begin{array}{l}\text { Коэффициент сжимаемости нефти, атм } \\
\text { Oil compressibility coefficient, } \text { atm }^{-1}\end{array}$ & $2,67 \cdot 10^{-4}$ & $9,86 \cdot 10^{-5}$ \\
\hline $\begin{array}{l}\text { Вязкость, } \mathrm{cП} \\
\text { Viscosity, } \mathrm{cP} \\
\end{array}$ & 0,54 & 0,54 \\
\hline $\begin{array}{l}\text { Атмосферное давление, ат } \\
\text { Atmosphere pressure, at }\end{array}$ & 1,033 & 1,033 \\
\hline \begin{tabular}{|l} 
Радиус скважины, м \\
Well radius, m
\end{tabular} & 0,108 & 0,108 \\
\hline $\begin{array}{l}\text { Коэффициент сжимаемости системы, атм } \\
\text { System compressibility coefficient, } \text { atm }^{-1} \\
\end{array}$ & $1,95 \cdot 10^{-4}$ & $1,95 \cdot 10^{-4}$ \\
\hline $\begin{array}{l}\text { Tемпература при нормальных условия }\left(+20{ }^{\circ} \mathrm{C}\right){ }^{\circ} \mathrm{K} \\
\text { Temperature, standard conditions }\left(+20^{\circ} \mathrm{C}\right){ }^{\circ} \mathrm{K}\end{array}$ & 293 & 293 \\
\hline $\begin{array}{l}\text { Дебит скважины до остановки, м }{ }^{3} / \text { сут } \\
\text { Well rate before shut-in, } \mathrm{m}^{3} / \text { day }\end{array}$ & 20 & 56 \\
\hline $\begin{array}{l}\text { Пористость } \\
\text { Porosity }\end{array}$ & 0,135 & 0,137 \\
\hline $\begin{array}{l}\text { Длина горизонтального ствола, м } \\
\text { Length of horizontal wellbore, } \mathrm{m}\end{array}$ & 136 & 235 \\
\hline $\begin{array}{l}\text { Экспертная оценка латеральной } \\
\text { проницаемости, мД } \\
\text { Expert of lateral permeability, mD }\end{array}$ & 1,2 & 1,2 \\
\hline $\begin{array}{l}\text { Время восстановления забойного давления до } \\
\text { пластового, Т, ч } \\
\text { Time, for which bottomhole pressure will restore } \\
\text { to reservoir pressure, T, hour }\end{array}$ & 600 & 550 \\
\hline
\end{tabular}

В табл. 3 приведены оценки латеральной проницаемости (10), пластового давления (11), скинфактора скважины (12) и полудлина трещины (13), полученные по аналогии с (10) адаптивным методом интегрированных моделей (АМИМ) (2)-(8) и с использованием ПО Saphir за разные периоды нестационарных исследований горизонтальных скважин.

$$
S_{n}^{*}=\frac{1}{2}\left(\frac{\alpha_{1, n}^{*}}{\alpha_{2, n}^{*}}-\ln \frac{k_{r, n}^{*}}{\phi \mu c_{t} r_{c}}\right),
$$




$$
x_{f, n}^{*}=\left(\frac{C_{s l} q B}{h \alpha_{2 l, n}^{*}}\right)^{2} \frac{\mu}{\phi c_{t} k_{y}},
$$

где $\alpha_{2 l, n}^{*}$ - оценки параметра линейного потока; $k_{y}=\frac{C_{s b r} q \mu B}{\alpha_{2 b r}^{*} h}\left(\frac{4}{L^{2} \phi \mu c_{t}}\right)^{0,36} ; \alpha_{2 b r}^{*}-$ оценки параметра бирадиального потока.

Таблица 3. Результаты нестационарных исследований горизонтальных скважин с трешинали гидроразыва пласта

Table 3. Results of transient tests in horizontal wells with hydraulic fractures

\begin{tabular}{|c|c|c|c|c|c|c|c|c|c|}
\hline \multirow[t]{2}{*}{ 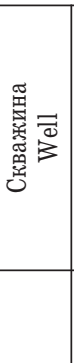 } & \multirow[t]{2}{*}{ 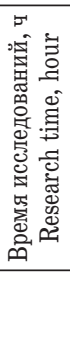 } & \multicolumn{2}{|c|}{$\begin{array}{c}\text { Латеральная } \\
\text { проница- } \\
\text { емость, мД } \\
\text { Lateral per- } \\
\text { meability, } \\
\text { mD }\end{array}$} & \multicolumn{2}{|c|}{$\begin{array}{c}\text { Пластовое } \\
\text { давление, } \\
\text { атм } \\
\text { Reservoir } \\
\text { pressure, } \\
\text { atm }\end{array}$} & \multicolumn{2}{|c|}{$\begin{array}{c}\text { Интеграль- } \\
\text { ный } \\
\text { скин-фактор } \\
\text { Total skin }\end{array}$} & \multicolumn{2}{|c|}{$\begin{array}{c}\text { Полудлина } \\
\text { трещин, м } \\
\text { Half-length } \\
\text { of fractures, } \\
\text { m }\end{array}$} \\
\hline & & .尹 & $\sum_{i}$ & .尹 & 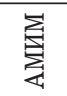 & 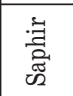 & $\sum_{i}$ & 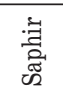 & $\sum_{i}$ \\
\hline \multirow{5}{*}{1} & 50 & 1,17 & 0,63 & 140,3 & 154,0 & $-4,63$ & $-5,41$ & 1,08 & 1,32 \\
\hline & 100 & 0.92 & 0,65 & 141,7 & 149,4 & $-4,78$ & $-5,54$ & 1,17 & 1,35 \\
\hline & 170 & 0,70 & 0,66 & 143,1 & 145,9 & $-4,89$ & $-5,67$ & 1,22 & 1,39 \\
\hline & 300 & 0,62 & 0,66 & 145,2 & 145,7 & $-5,08$ & $-5,67$ & 1,28 & 1,42 \\
\hline & 479 & 0,60 & 0,66 & 146,9 & 145,1 & $-5,36$ & $-5,61$ & 1,39 & 1,44 \\
\hline \multirow{6}{*}{2} & & 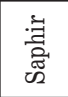 & $\sum_{i}$ & : & $\sum_{\text {窟 }}$ & : & $\sum_{i}$ & $\begin{array}{l}\text { 吉 } \\
\text { 离 } \\
\text { की }\end{array}$ & $\sum_{i}$ \\
\hline & 50 & 1,02 & 0,61 & 128,9 & 145,5 & $-4,56$ & $-5,0$ & 15,3 & 20,1 \\
\hline & 100 & 0,87 & 0,63 & 130,1 & 137,4 & $-4,98$ & $-5,13$ & 17,1 & 21,3 \\
\hline & 160 & 0,66 & 0,63 & 130,7 & 134,3 & $-5,15$ & $-5,20$ & 20,5 & 23,5 \\
\hline & 300 & 0,58 & 0,63 & 132,1 & 133,8 & $-5,43$ & $-5,23$ & 24,5 & 23,4 \\
\hline & 456 & 0,58 & 0,64 & 133,7 & 133,5 & $-5,43$ & $-5,24$ & 24,5 & 23,4 \\
\hline
\end{tabular}

\section{СПИСОК ЛИТЕРАТУРЫ}

1. Кременецкий М.И., Ипатов А.И., Гуляев Д.Н. Информационное обеспечение и технологии гидродинамического моделирования нефтяных и газовых скважин. - М.; Ижевск: Институт компьютерных исследований, 2012. - 896 с.

2. Bourdet D., Ayoub J.A., Pirard Y.M. Use of pressure derivative in well test interpretation // Society of Petroleum Engineers. 1984. - № 12777. - P. 293-302.

3. Хисамов Р.С., Сулейманов Э.И., Фархуллин Р.Г. Гидродинамические исследования скважин и методы обработки результатов измерений. - М.: ОАО «ВНИИОЭНГ», 2000. - 228 с.

4. Ozkan E., Brown M., Raghavan R. Comparison of fractured horizontal-well performance in conventional and unconventional reservoirs // Society of Petroleum Engineers. - 2009. № 121290, - P. 1-16.

5. Развитие геофизического и гидродинамического мониторинга на этапе перехода к разработке объектов с трудноизвлекаемыми запасами нефти / В.Г. Мартынов, А.И. Ипатов, М.И. Кременецкий, Д.Н. Гуляев, В.М. Кричевский, В.В. Кокурина, С.И. Мельников // Нефтяное хозяйство. - 2014. - № 3. C. $106-109$.

6. Horne R.N., Temeng K.0. Relative productivities and pressure transient modeling of horizontal wells with multiple fractures //
Из табл. 3 видно, что рассмотренный метод идентификации фильтрационных потоков АМИМ (2)-(5) позволяет получить оценки латеральной проницаемости, пластового давления, скин-фактора скважины и длину трещины ГРП на более коротких недовосстановленных кривых забойного давления по сравнению с оценками, полученными с использованием ПК Saphir. Например, для скважины № 1 стабилизация оценок латеральной проницаемости, полученных методом АМИМ, наступает через 100 часов нестационарных исследований. Стабилизация оценок латеральной проницаемости, полученных в ПК Saphir, наступает значительно позже, через 480 часов исследований.

\section{Выводы}

Для решения задачи идентификации фильтрационных потоков в процессе нестационарных исследований горизонтальных скважин с трещинами гидроразрыва пласта предложено использовать интегрированные системы моделей кривой восстановления забойного давления с нестационарными параметрами, с учетом и корректировкой дополнительной информации.

На примерах обработки результатов нестационарных исследований двух горизонтальных скважин с трещинами гидроразрыва пласта нефтяного месторождения показано, что предложенные модели и алгоритмы идентификации фильтрационных потоков позволяют:

1) определять латеральную проницаемость, пластовое давление, полудлину трещин, скин-фактор скважины в процессе проведения нестационарных исследований по восстановлению забойного давления в условиях отсутствия участка позднего радиального потока;

2) значительно сократить время простоя скважин.

Society of Petroleum Engineers. - 1995. - № 29891. P. 563-574.

7. Кременецкий М.И., Кокурина В., Морозовский Н. Оценка добычных возможностей пластов низкой проницаемости вскрытых трещинами гидроразрыва сложной конфигурации по результатам ГДИС // Society of Petroleum Engineers. -2017. № 187766-RU. - C. 1-19.

8. Kuchuk F.J., Onur M., Hollaender F. Pressure transient formation and well testing: Convolution, Deconvolution and Nonline Estimation. - Amsterdam: Elsevier, Developments in Petroleum Science, 2010. - V. 57. - 389 p.

9. Brown M., Ozkan E., Raghavan R. Practical solutions for pressure-transient responses of fractured horizontal wells in unconventional shale reservoirs // Society of Petroleum Engineers. 2011. - № 125043. - P. 663-676.

10. Raghavan R., Chen C., Bijan A. An analysis of horizontal wells intercepted by multiple fractures / Society of Petroleum Engineers. - 1997. - № 27652. - P. 235-245.

11. Сергеев В.Л., Донг Ван Хоанг. Адаптивная интерпретация гидродинамических исследований горизонтальных скважин с идентификацией псевдорадиального потока // Известия Томского политехнического университета. Инжиниринг георесурсов. -2017. - Т. 328. - № 10. - С. 67-73. 
12. Zerzar A., Tiab D., Bettam Y. Interpretation of multiple hydraulically fractured horizontal wells / Society of Petroleum Engineers. - 2004. - № 88707. - P. 1-13.

13. Biryukov D., Kuchuk F.J. Pressure transient behavior of horizontal wells intersecting multiple hydraulic fractures in naturally fractured reservoirs // Transport in Porous Media. - 2015. № 110. - P. 369-408.

14. Kuchuck F.J., Habashy T. Pressure behavior of horizontal wells with multiple fractures // Society of Petroleum Engineers. 1994. - № 27971. - P. 1-11.

15. Geilikman M., Wong S. Hydraulic fractures interference in horizontal wells: Compact and diffuse patterns of fracture growth // Society of Petroleum Engineers. - 2013. - № 167161-MS. P. 1-13.

16. Larsen L., Hegre T. Pressure transient analysis of multifractured horizontal wells // Society of Petroleum Engineers. - 1994. № 28389. - P. 265-276.

\section{Информация об авторах}

Сергеев В.Л., доктор технических наук, профессор отделения нефтегазового дела Школы природных ресурсов Национального исследовательского Томского политехнического университета.

Донг Ван Хоанг, аспирант отделения нефтегазового дела Школы природных ресурсов Национального исследовательского Томского политехнического университета.
17. Сергеев В.Л., Ву К.Д. К оптимизации адаптивных алгоритмов идентификации и интерпретации гидродинамических исследований с учетом влияния ствола скважины // Доклады ТУСУРа. - Март 2016. - № 1 (39). - С. 98-102.

18. Sergeev V.L., Dong Van Hoang. Adaptive identification and interpretation of pressure transient tests of horizontal wells: Challenges and perspectives / Proc. IOP Conference Series: Earth and Environmental Science. - 2016. - V. 43. - № 1. - P. 1-6.

19. Сергеев В.Л. Интегрированные системы идентификации. Томск: Изд-во Томского политехнического университета, 2011. $-198 \mathrm{c}$

20. Пантелеев А.В., Летова Т.А. Методы оптимизации в примерах и задачах. - СПб.: Издательство «Лань», 2015. - 512 с.

Поступила 27.12.2018 2. 


\title{
IDENTIFICATION OF FILTRATION FLOW REGIMES IN HYDRODYNAMIC STUDIES OF HORIZONTAL WELLS WITH HYDRAULIC FRACTURES
}

\author{
Viktor L. Sergeev', \\ SergeevVL@ignd.tpu.ru \\ Dong Van Hoang', \\ hoang.tpu@gmail.com \\ National Research Tomsk Polytechnic University,
30, Lenin Avenue, Tomsk, 634050, Russia.
}

The relevance of the research is caused by the need to create methods for operational processing the results of non-stationary researches in horizontal wells with hydraulic fractures, and increasing accuracy of parameter estimates of oil reservoirs and decreasing shut-in time.

The main aim of the research is to develop the models and algorithms for identifying filtration flow regimes, defining filtration characteristics and reservoir pressure in processing of non-stationary researches in horizontal wells with hydraulic fractures.

The methods are based on the researches in the field of hydrodynamic studies of wells with hydraulic fractures system analysis, system modeling taking into account additional information and expert estimates, function optimization, linear algebra. Using integral system of wellbore pressure models with time-dependent variables, with account of additional prior information and expert estimates about reservoir permeability and reservoir pressure, the problem of filtration flow identification was solved. Testing of models and algorithms for flow identification and determination of reservoir and fracture parameters was carried out on the basis of field data from non-stationary researches of horizontal oil wells with hydraulic fractures by pressure buildup curves using the software package Saphir.

The results. Processing non-stationary research results by pressure buildup curves of horizontal wells shows that the proposed models and algorithms for identification of filtration flow regimes allow determining the effective length of horizontal wellbore, lateral permeability, reservoir pressure and skin factor in the process of research, under conditions of partial or complete absence of late radial flow on pressure buildup curves, significantly reducing shut-in time.

\section{Key words:}

Non-stationary researches, filtration flow regimes, identification, adaptation, wellbore pressure,

horizontal wells, oil reservoirs, hydraulic fracturing.

\section{REFERENCES}

1. Kremenetsky M.I., Ipatov A.I., Gulyaev D.N. Informatsionnoe obespechenie i tekhnologii gidrodinamicheskogo modelorovaniya neftyanykh $i$ gazovykh zalezhey [Information and technology of hydrodynamic modeling of oil and gas deposits]. Moscow; Izhevsk, Institute of computer research Press, 2012.896 p.

2. Bourdet D., Ayoub J.A., Pirard Y.M. Use of pressure derivative in well test interpretation. Society of Petroleum Engineers, 1989, no. 12777 , pp. 293-302.

3. Khisamov R.S., Suleymanov E.I., Farkhullin R.G. Gidrodina micheskie issledovaniya skvazhin i metody obrabotki rezultatov izmereny [Hydrodynamic studies of wells and methods of processing the results of measurements]. Moscow, VNIIOENG Publ., 2000. $228 \mathrm{p}$.

4. Ozkan E., Brown M., Raghavan R. Comparison of fractured horizontal-well performance in conventional and unconventional reservoirs. Society of Petroleum Engineers, 2009, no. 121290, pp. 1-16.

5. Martynov V.G., Ipatov A.I., Kremenetskiy M.I., Gulyaev D.N., Krichevskiy V.M., Kokurina V.V., Melnikov S.I. Permanent reservoir monitoring by logging gages at the stage of tight oil recovery. Neftyanoe khozyaystvo, 2014, no. 1, pp. 106-109. In Rus.

6. Horne R.N., Temeng K.0. Relative productivities and pressure transient modeling of horizontal wells with multiple fractures. Society of Petroleum Engineers, 1995, no. 29891, pp. 563-574.

7. Kremenetskiy M.I., Kokurina V., Morozovskiy N. PI Evaluation by Well Tests in Case of Low Permeability Formations Exposed by Complex Geometry Fracs. Society of Petroleum Engineers, 2017, no. 187766-RU, pp. 1-19. In Rus.

8. Kuchuk F.J., Onur M., Hollaender F. Pressure transient formation and well testing: Convolution, Deconvolution and Nonline Estimation. Amsterdam, Elsevier, Developments in Petroleum Science, 2010. Vol. 57, 389 p.
9. Brown M., Ozkan E., Raghavan R. Practical solutions for pressure-transient responses of fractured horizontal wells in unconventional shale reservoirs. Society of Petroleum Engineers, 2011, no. 125043 , pp. 663-676.

10. Raghavan R., Chen C., Bijan A. An analysis of horizontal wells intercepted by multiple fractures. Society of Petroleum Engineers, 1997, no. 27652, pp. 235-245.

11. Sergeev V.L., Dong Van Hoang. Adaptive interpretation of pressure transient tests of horizontal wells with pseudoradial flow identification. Bulletin of the Tomsk Polytechnic University. Geo Assets Engineering, 2017, vol. 328, no. 10, pp. 67-73. In Rus.

12. Zerzar A., Tiab D., Bettam Y. Interpretation of multiple hydraulically fractured horizontal wells. Society of Petroleum Engineers, 2004, no. 88707, pp. 1-13.

13. Biryukov D., Kuchuk F.J. Pressure transient behavior of horizontal wells intersecting multiple hydraulic fractures in naturally fractured reservoirs. Transport in Porous Media, 2015, no. 110 , pp. $369-408$.

14. Kuchuck F.J., Habashy T. Pressure behavior of horizontal wells with multiple fractures. Society of Petroleum Engineers, 1994, no. 27971, pp. 1-11.

15. Geilikman M., Wong S. Hydraulic fractures interference in horizontal wells: Compact and diffuse patterns of fracture growth. Society of Petroleum Engineers, 2013, no. 167161-MS, pp. 1-13.

16. Larsen L., Hegre T. Pressure transient analysis of multifractured horizontal wells. Society of Petroleum Engineers, 1994, no. 28389 , pp. $265-276$.

17. Sergeev V.L., Vu Q.D. Optimization of adaptive algorithms for identification and interpretation of hydrodynamics research considering wellbore storage. Doklady TUSUR, 2016, March, no. 1 (39), pp. 98-102. In Rus. 
18. Sergeev V.L., Dong Van Hoang. Adaptive identification and interpretation of pressure transient tests of horizontal wells: Challenges and perspectives. Proc. IOP Conference Series: Earth and Environmental Science, 2016, vol. 43, no. 1, pp. 1-6.

19. Sergeev V.L. Integrirovannye sistemy identifikatsii [Integrated identification system]. Tomsk, Tomsk Polytechnic University Publ. house, 2011.198 p.

\section{Information about the authors}

Viktor L. Sergeev, Dr. Sc., professor, National Research Tomsk Polytechnic University.

Dong Van Hoang, ostgraduate, National Research Tomsk Polytechnic University.
20. Panteleev A.V., Letova T.A. Metody optimizatsii v primerakh $i$ zadachakh [Optimization methods in examples and tasks]. St.-Petersburg, Lan Publ., 2015. 512 p.

Received: 27 December 2018. 